\title{
Hacia un método de transferencia de aprendizaje en señales de EEG de habla imaginada
}

\author{
Jessica Nayeli López Espejel ${ }^{1}$, Maya Carrillo Ruíz ${ }^{1}$, Luis Villaseñor Pineda ${ }^{2}$, \\ Alejandro Torres García ${ }^{2}$ \\ ${ }^{1}$ Benemérita Universidad Autónoma de Puebla, Puebla, México \\ ${ }^{2}$ Instituto Nacional de Astrofísica, Óptica y Electrónica, Puebla, México \\ acissejol@hotmail.com,crrllrzmy@gmail.com,luis.villasenor.pineda@gmail.com, \\ alejandro.torres@ccc.inaoep.mx
}

\begin{abstract}
Resumen. El fin último de este trabajo es disminuir o eliminar la etapa de entrenamiento, para cuando un nuevo sujeto utilice una BCI (brain-computer interface) basada en habla imaginada. La etapa de entrenamiento se debe a que es necesario adquirir suficiente información para identificar los patrones que permitan distinguir lo que el sujeto imagina pronunciar. Ahora bien, en habla imaginada, como cualquier otro potencial evocado, el proceso de entrenamiento es tardado y tedioso. En este artículo se presenta una serie de experimentos que busca comprobar si su información de entrenamiento ya disponible para un grupo de sujetos puede aprovecharse para un nuevo sujeto. El método empleado usa mapas auto- organizados para seleccionar la información ya disponible con la cual se generan clasificadores binarios para identificar las palabras presentes en los EEG del nuevo sujeto. Los resultados alcanzados son alentadores y dan pauta para el diseño de un método apropiado para transferencia de aprendizaje sujetoa-sujeto en habla imaginada.
\end{abstract}

Palabras clave: transferencia de aprendizaje, habla imaginada, EEG, random forest.

\section{Towards a Method of Transfer Learning in Imagined Speech EEG Signals}

\begin{abstract}
The target of this research is decrease or eliminate the training stage, when a new subject uses a brain- computer interface (BCI) based on imagined speech. In the training phase it is necessary to acquire enough information to identify the patterns that allow to distinguish what the subject imagines to pronounce. Then, in imagined speech, like any other evoked potential, the training process is tedious. This article presents experiments that seek to verify if the training information and the available information for a group of subjects can be used for a new person. The proposed method uses self- organized maps to select information in order to generated binary classifiers to identify the words presented in a new EEG subject. The results are encouraged and give the
\end{abstract}


guideline to design a suitable method for a subject to subject transfer learning method for imagined speech.

Keywords: transfer learning, imagined speech, EEG, random forest.

\section{Introducción}

Durante las últimas dos décadas, una variedad de métodos han evaluado la posibilidad de transformar las señales cerebrales obtenidas a través del cráneo y el cuero cabelludo para activar diferentes dispositivos. De esta forma surge una nueva interfaz que no requiere ser manipulada a través de actividades musculares. Este tipo de interfaz recibe el nombre de BCI (Brain-Computer Interface, en español Interfaz CerebroComputadora). En breve, una BCI es un sistema cuyo objetivo es establecer la comunicación entre un humano y un sistema automático, a través de las señales cerebrales, las cuales pueden ser detectadas a través de diferentes instrumentos [1].

Las BCI utilizan diferentes fuentes electrofisiológicas, tales como: los 2 ritmos sensoriales motrices (mu y beta) y los potenciales evocados. Bajo estos últimos cae el caso del habla imaginada, también referida como habla interna o habla no pronunciada. Este término se refiere a imaginar la pronunciación de una sílaba o palabra sin emitir sonidos ni articular gestos para ello [2].

Las BCIs en general presentan dos grandes problemas: (i) el usuario debe pasar por una fase de entrenamiento que en ocasiones puede llevar días o incluso meses, y (ii) las bajas tasas de comunicación, es decir, comunicar un comando puede llevarse mucho tiempo, por ejemplo, en el caso de las BCI basadas en P300 comunicar una sola palabra procesada, puede llevarse un minuto.

Una BCI basada en habla imaginada disminuye de forma importante las tasas de comunicación, y aunque la fase de entrenamiento es menor ésta sigue siendo importante. De ahí el interés en aplicar transferencia de aprendizaje en esta tarea, para disminuir el tiempo dedicado por un usuario en la etapa de entrenamiento. La transferencia de aprendizaje se describe como el procedimiento de usar los datos de una tarea para aumentar el rendimiento de una tarea relacionada. [3]

La transferencia de aprendizaje en BCI puede aplicarse en diferentes escenarios, dos de ellos son: de sujeto-a-sujeto y de sesión-a-sesión. En ambas situaciones existen variaciones de muy diversa naturaleza. Éstas van desde una leve desigualdad en colocación de los electrodos por la forma de la cabeza, el contacto del cuero cabelludo con los electrodos es distinto, además de diferencias debidas al estado anímico de las personas, le cual puede cambiar de un día a otro, su concentración puede verse alterada, etc.

En el presente trabajo se muestra los primeros pasos hacia un método transferencia de aprendizaje sujeto-a-sujeto. En este caso, observamos si los datos de entrenamiento adquiridos para otros sujetos pueden ser utilizados como datos de entrenamiento para un nuevo sujeto. La tarea no es simple pues además de las variaciones mencionadas, cuando se trabaja con habla imaginada, no se tiene la certeza de las regiones cerebrales que intervienen, y es claro que cada sujeto produce distintas señales cerebrales, aunque se trate de la misma palabra. 
El resto del trabajo está organizado de la siguiente manera: en la Sección 2 se presenta el trabajo relacionado, en la Sección 3 se describe el método propuesto, en la Sección 4 los experimentos y resultados obtenidos, y finalmente en la Sección 5 las conclusiones y el trabajo a futuro.

\section{Trabajo relacionado}

La transferencia de aprendizaje en la problemática de las BCI basadas en EEG puede aplicarse desde diferentes perspectivas. El escenario más frecuente es el tratar de adaptar una BCI a un nuevo sujeto a partir de datos previos registrados con otros sujetos. Sin embargo, la transferencia de aprendizaje también puede aplicarse a un mismo sujeto para abordar el problema de la habituación (i.e. la variabilidad de la señal cerebral entre diferentes sesiones) o para incrementar el vocabulario de palabras imaginadas a reconocer para el caso del habla imaginada. A continuación se presentan algunos trabajos que han aplicado transferencia de aprendizaje en BCI. Cabe señalar que hasta donde sabemos esta técnica no ha sido aplicada a habla imaginada.

En [4] la base de datos que contiene los EEG es de tareas de movimientos imaginarios de la mano derecha y pie derecho, y está formada por 5 sujetos. En este artículo presentaron una modificación del algoritmo de Patrones Espaciales Comunes CSP (Common Spatial Pattern) para la transferencia de aprendizaje de sujeto a sujeto, dicha modificación consistió en una combinación lineal de matrices de covarianza de los individuos a los que se les tomo el EEG. Además, también desarrollaron dos métodos para determinar una matriz de covarianza compuesta, que es una suma ponderada de las matrices de covarianza. En el primer método se ajusta el parámetro $\lambda$ $[0,1]$ para ajustar la matriz de covarianza. En el segundo método se enfatiza en las matrices de covarianza para los sujetos con características similares, por lo que se calcula la divergencia entre las distribuciones de datos para dos o más sujetos que se estén comparando. La precisión de clasificación media para los sujetos en el primer método es de 75.23 y para el segundo método propuesto es de 82.5653 , cuyos resultados son mejores que en el método tradicional, puesto que para dicho método la precisión de clasificación media en los sujetos es de 74.16.

En [5] presentaron un estudio de las señales EEG de niños con déficit de atención e hiperactividad con el propósito de apoyar un sistema de diagnóstico asistido por computadora. Las señales EEG se tomaron a 13 sujetos, y de ellos la mitad estaba diagnosticado medicamente con TDAH (por sus siglas, Trastorno de Déficit de Atención e Hiperactividad) y la otra mitad estaban clínicamente sanos. Los EEG se analizaron con la Transformada Discreta de Wavelet para extraer las características del espectro de potencia de la señal cerebral, y una técnica de umbral fue utilizada para mejorar aún más la calidad de los datos debido a que tenían ruido de artefactos. Para discriminar el nivel de atención en la actividad eléctrica cerebral de los niños con TDAH, se utilizaron mapas auto- organizados cuya entrada eran los coeficientes obtenidos de la TDW. La transferencia de aprendizaje se utiliza para discernir entre los niños que tienen TDAH y los que no, a través de las mejores señales, agrupadas por la SOM. 
En [6] propusieron un algoritmo para reducir el tiempo de adaptación (o calibración) para un sujeto nuevo. Debido a la variabilidad de sujetos, por lo general un nuevo individuo tiene que someterse a una sesión de 30 minutos de calibración para poder recuperar datos suficientes para la formación de un modelo BCI basado en sus patrones/cerebro. El algoritmo propuesto consta de dos pasos: en el primer paso se adaptan los datos del nuevo sujeto a los datos de los EEG's que ya se tienen en la base de datos; en el segundo paso se selecciona el EEG del individuo de la base de datos que tenga más coincidencias con los datos del EEG del nuevo sujeto.

La base de datos utilizada contiene EEG's de 9 sujetos, y para cada uno se emplearon 22 electrodos. La base de datos tiene EEG de cuatro actividades imaginarias: mano derecha, mano izquierda, lengua y pie; sin embargo, para este trabajo únicamente se utilizaron dos tipos de movimiento imaginado: mano derecha y mano izquierda. En la etapa experimental de los nuevos sujetos, fueron 8 los individuos con los que se trabajó. De acuerdo a los resultados obtenidos, se puede apreciar que el algoritmo propuesto en este trabajo de investigación reduce el tiempo de calibración en un $85 \%$.

En [7] están conscientes de la problemática que existe entre la variabilidad de las señales cuando se toman de sujeto-a-sujeto y de sesión-a-sesión, por lo cual propusieron lo siguiente: 1) un método para la extracción de diccionarios compartido por múltiples sujetos, mediante el empleo de una técnica de diccionario de procesamiento digital de señales, modificada para compensar las variaciones entre sujetos y sesiones; 2) un enfoque de decodificación de transferencia, en donde los sujetos se encuentran en estado de reposo, y de esta forma eliminan la necesidad de una calibración estándar. La aplicación de la metodología que los autores proponen a un conjunto de datos de EEG's durante una tarea de atención visual- espacial, muestran que la compensación de variabilidad se logra estando los sujetos en estado de reposo, y de esa manera se reduce la redundancia en los diccionarios.

La aplicación de la decodificación de transferencia de conocimiento en los sujetos logró un mejor desempeño frente a los métodos existentes de decodificación. Por lo tanto, los resultados revelan que el análisis de las actividades cerebrales con el método propuesto permite reducir el tiempo de calibración, y es muy útil para el uso práctico de BCI en ambientes variables.

En [3] desarrollaron un framework para la transferencia de aprendizaje utilizando una BCI. Este trabajo consiste de dos experimentos, el primer experimento se realizó tomado el EEG de 10 sujetos sanos (2 mujeres y 8 hombres de entre 25.5 y 25.6 años), el procedimiento constó de un total de 150 ensayos por clase (derecha/ izquierda) y por persona. La extracción de características se llevó a cabo a través de filtros espaciales usando una configuración de superficie de Laplace y también empleando la Transformada Discreta de Fourier.

Para la clasificación de las actividades mentales se utilizaron cinco algoritmos (dos de aprendizaje multitarea denominados: MT_FD y MT por sus siglas en inglés "multitask learning", un algoritmo de cresta de regresión estándar, otro de cresta de regresión pero usando FD, y por último máquinas de soporte vectorial), y entre ellos, el que tuvo la mayor precisión de clasificación aplicando la transferencia de aprendizaje fue el algoritmo MT_FD con el 65\% de precisión. 


\section{Método propuesto}

El método para realizar nuestros primeros experimentos hacia la trasferencia de aprendizaje consiste de cinco pasos: pre- procesamiento de la señal, caracterización de las señales, selección del conjunto de entrenamiento, entrenamiento de clasificadores binarios y evaluación con nuevos sujetos. Las subsecciones a continuación detallan cada uno de estos pasos.

\subsection{Pre-procesamiento}

El pre-procesamiento de la señal de los EEG se llevó a cabo con el método de referencia promedio (CAR, por sus siglas en inglés). El propósito de este método es mejorar la señal aprovechando que se tienen lecturas simultáneas de los electrodos. La fórmula que lo describe, es la siguiente:

$$
\mathbf{V}_{\mathbf{i}}^{\mathrm{CAR}}=\mathbf{V}_{\mathbf{i}}^{\mathrm{ER}}-\frac{1}{\mathbf{n}} \sum_{\mathbf{j}=1}^{\mathrm{n}} \mathbf{V}_{\mathbf{j}}^{\mathrm{ER}}
$$

donde $\mathbf{V}_{\mathbf{j}}^{\mathbf{E R}}$ es el potencial entre el i- ésimo electrodo y la referencia, y n es el número de electrodos.

\subsection{Caracterización de las señales}

En este paso se extraen características de las señales utilizando la Transformada Discreta de Wavelet (DWT). La Transformada Discreta Wavelet se utilizó con 4 niveles de descomposición ya que sólo son de interés los rangos de frecuencia menores a 32 Hz. Se empleó la wavelet biortogonal 2.2 de la toolbox de Matlab R2013b ya que de acuerdo a [2] es la familia de wavelet que genera los mejores resultados. De estos 4 niveles, se obtienen coeficientes de aproximación y coeficientes de detalle, con las siglas cA y cD, tal como se muestra en la Figura 1. Posteriormente, se calculó la energía instantánea entre los 4 niveles.

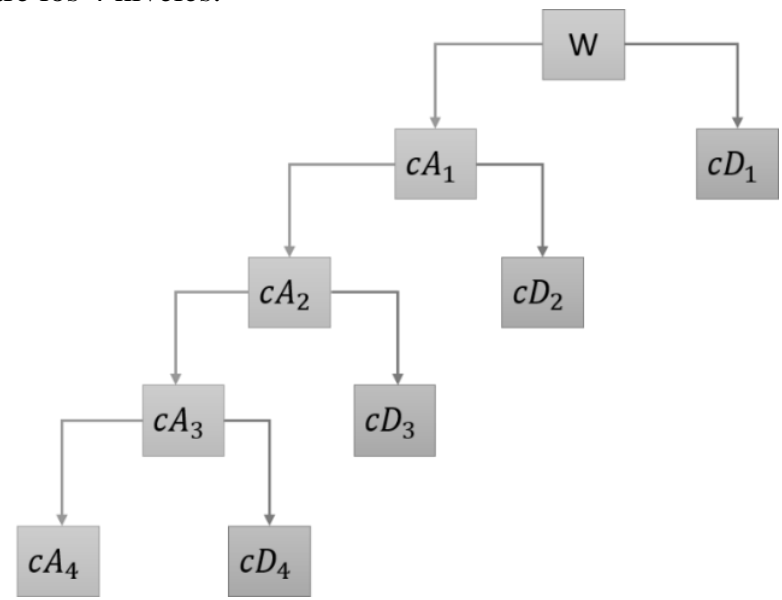

Fig. 1. Descomposición wavelet en 4 niveles. 
La energía instantánea se obtiene a partir de los coeficientes calculados con la DWT. De esta forma, cada repetición de cada una de las palabras imaginadas se representó mediante 5 valores que representan la diferencia de energía de cada uno de los niveles de descomposición (D1-D4 y A4). Gracias a este procedimiento se obtiene el mismo número de coeficientes para todas las ventanas de la señal. Este proceso de extracción se realiza para cada electrodo.

Una vez caracterizadas las señales mediante la energía instantánea, se procedió a entrenar clasificadores binarios como primer paso para el proceso de transferencia de aprendizaje.

\subsection{Entrenamiento de clasificadores binarios}

En este paso se crearon clasificadores binarios para las combinaciones de las palabras imaginadas arriba, abajo, derecha, izquierda. Se decidió hacer clasificadores binarios para observar la tasa de transferencia para cada par de palabras. A pesar de que se sabe que existen diferencias entre sujetos a pesar de imaginar la misma palabra, deben existir ciertas semejanzas pues se evoca el movimiento de aparato fonador para producir sonidos que corresponden a cierta norma. Así para la palabra arriba deben existir semejanzas en las señales producidas por diferentes sujetos.

Sin embargo, el grado de semejanza puede variar mucho de un sujeto a otro, de ahí que se crearon clasificadores binarios considerando los datos de entrenamiento de 10 sujetos. Para crear estos clasificadores base se seleccionaron aquellas señales más semejantes entre los 10 sujetos. Para ello se utilizó un algoritmo de clustering en nuestro caso se utilizaron mapas auto-organizados (SOM por sus siglas en inglés). Los elementos agrupados de manera correcta por la SOM, se tomaron como datos de entrenamiento para generar cada uno de los clasificadores base.

Los clasificadores binarios consideran todas las posibles combinaciones de las palabras como en [6], entrenados con las instancias agrupadas de manera correcta por la SOM.

\subsection{Evaluación en nuevos sujetos}

En este paso se evaluaron los clasificadores binarios con los datos de las cuatro palabras imaginadas de un conjunto de sujetos diferente. De esta forma se calculó el porcentaje de instancias clasificadas correctamente para estos nuevos sujetos. Es decir, el porcentaje de transferencia realizado de manera correcta.

\section{Experimentos y resultados}

\section{Base de datos}

La base de datos empleada es la descrita en [2]. Para la elaboración de esta base de datos se utilizó el kit de EMOTIV. Las muestras de EEG se le practicaron a 27 sujetos, cada individuo repitió 33 veces (en algunos casos más repeticiones) 5 palabras (arriba, abajo, derecha, izquierda, seleccionar). De manera gráfica, la base de datos se representa como muestra la Figura 2. 


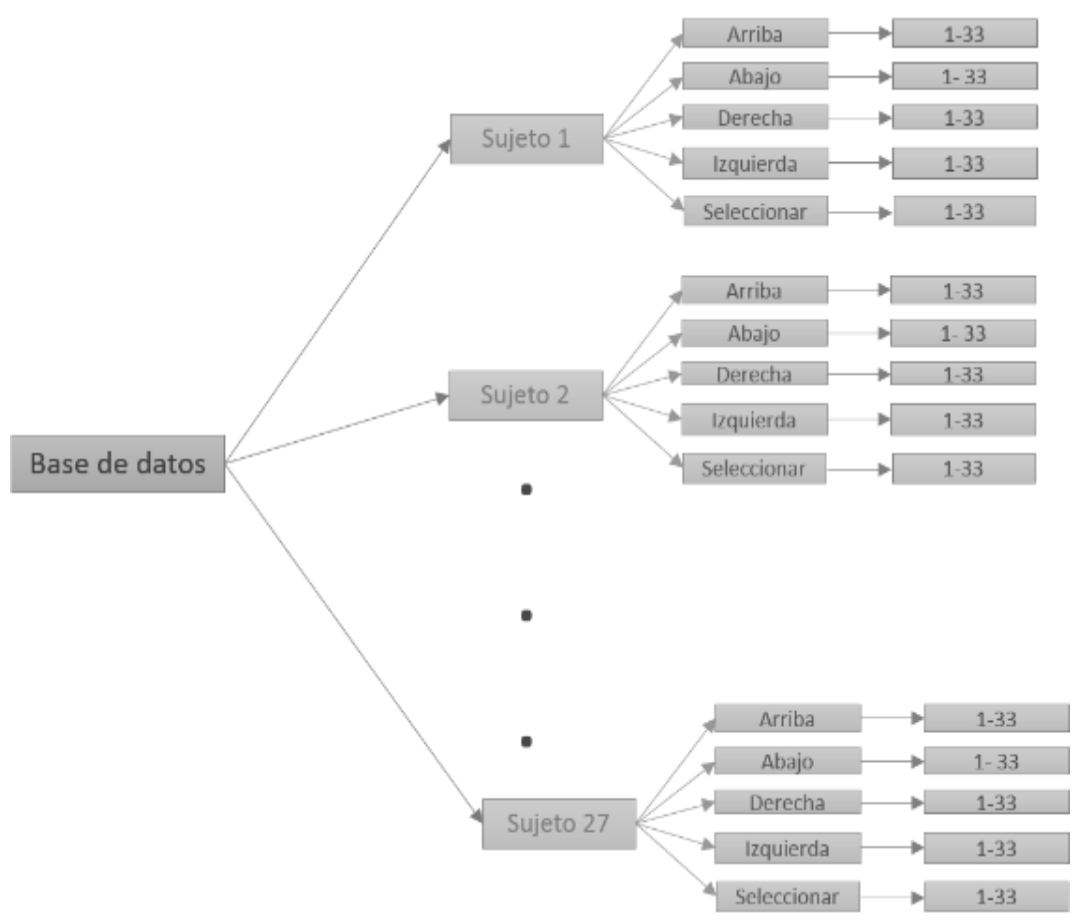

Fig. 2. Base de datos.

Cabe mencionar que la palabra "seleccionar" presente en la base de datos se excluyó para este trabajo. Las SOMs fueron implementadas en Matlab 2015a utilizando la toolbox y los clasificadores binarios se implementaron en Weka empleando Random Forest, con las instancias agrupadas de manera correcta por la SOM. Cabe señalar que para construir los clasificadores binarios los datos fueron balanceados usando undersampling.

A partir de estos clasificadores, se evaluó la transferencia de aprendizaje al clasificar las señales de nuevos sujetos. Los resultados obtenidos de cada uno de los clasificadores binarios se presentan en las tablas 3 y 4 .

Como primera observación de las tablas 3 y 4 , nuestros resultados muestran tasas de exactitud promedio entre $53 \%$ y $60 \%$. Estos resultados son muy alentadores pues hay que recordar que estos resultados se han obtenido sin hacer ningún tipo de adaptación hacia los nuevos sujetos. Lo que es más, las clases más diferenciables alcanzan exactitudes promedio de $60.07 \%$ y $59.18 \%$ (clasificadores arriba-abajo y derechaizquierda), alcanzando rendimientos similares a los reportados en el estado del arte [5].

Observando a detalle los resultados, algunos sujetos alcanzan resultados muy altos, a pesar de que para otros los resultados no son favorables. Por ejemplo, en el caso del sujeto número 22 en todas las combinaciones que incluyen la clase arriba presenta altas exactitudes (arriba-abajo $=90.91 \%$, arriba-derecha $=89.39 \%$ y arriba-izquierda $=$ $83.33 \%$ ). De igual manera, el sujeto 26 para todas las combinaciones su exactitud va de $57.58 \%$ a $89.39 \%$, seguido del sujeto 3 , cuyo porcentaje de exactitud en la 
transferencia esta entre $59.09 \%$ y $66.67 \%$ en cinco de seis combinaciones entre las clases.

Tabla 1. Resultado de tres combinaciones para 17 sujetos nuevos.

\begin{tabular}{|c|c|c|c|c|c|c|c|c|c|}
\cline { 2 - 10 } \multicolumn{2}{|c|}{} & \multicolumn{2}{|c|}{ ARRIBA-ABAJO } & \multicolumn{2}{|c|}{ ARRIBA-DERECHA } & \multicolumn{2}{|c|}{ ARRIBA-IZQUIERDA } \\
\hline $\begin{array}{c}\text { Sujet } \\
\text { o }\end{array}$ & EXAC. & $\begin{array}{c}\text { Precisió } \\
\text { n } \\
\text { arriba }\end{array}$ & $\begin{array}{c}\text { Precisió } \\
\text { n } \\
\text { abajo }\end{array}$ & EXAC. & $\begin{array}{c}\text { Precisió } \\
\text { n } \\
\text { arriba }\end{array}$ & $\begin{array}{c}\text { Precisió } \\
\text { n } \\
\text { derecha }\end{array}$ & EXAC. & $\begin{array}{c}\text { Precisió } \\
\text { n } \\
\text { arriba }\end{array}$ & $\begin{array}{c}\text { Precisió } \\
\text { n } \\
\text { izquierd } \\
\text { a }\end{array}$ \\
\hline 2 & $50.00 \%$ & 0.50 & 0.00 & $50.00 \%$ & 0.500 & 0.000 & $48.48 \%$ & 0.492 & 0.400 \\
\hline 3 & $63.64 \%$ & 0.636 & 0.636 & $62.12 \%$ & 0.645 & 0.580 & $66.67 \%$ & 0.900 & 0.620 \\
\hline 6 & $48.48 \%$ & 0.400 & 0.492 & $48.48 \%$ & 0.333 & 0.492 & $50.00 \%$ & 0.500 & 0.500 \\
\hline 7 & $56.06 \%$ & 0.534 & 0.750 & $45.45 \%$ & 0.471 & 0.400 & $53.03 \%$ & 0.531 & 0.529 \\
\hline 10 & $63.64 \%$ & 0.636 & 0.636 & $62.12 \%$ & 0.633 & 0.611 & $48.48 \%$ & 0.486 & 0.484 \\
\hline 12 & $62.12 \%$ & 0.912 & 0.645 & $51.52 \%$ & 0.700 & 0.512 & $56.06 \%$ & 0.900 & 0.652 \\
\hline 13 & $50.00 \%$ & 0.500 & 0.500 & $51.52 \%$ & 1.000 & 0.508 & $51.52 \%$ & 1.000 & 0.508 \\
\hline 14 & $45.45 \%$ & 0.455 & 0.455 & $53.03 \%$ & 0.589 & 0.519 & $48.48 \%$ & 0.455 & 0.491 \\
\hline 16 & $63.64 \%$ & 0.588 & 0.714 & $63.64 \%$ & 0.700 & 0.610 & $51.52 \%$ & 0.714 & 0.645 \\
\hline 17 & $62.12 \%$ & 0.571 & 0.900 & $63.64 \%$ & 0.585 & 0.846 & $66.67 \%$ & 0.604 & 0.923 \\
\hline 19 & $48.48 \%$ & 0.492 & 0.429 & $53.03 \%$ & 0.519 & 0.583 & $50.00 \%$ & 0.500 & 0.500 \\
\hline 21 & $51.52 \%$ & 0.788 & 0.182 & $51.52 \%$ & 0.509 & 0.538 & $56.06 \%$ & 0.540 & 0.625 \\
\hline 22 & $90.91 \%$ & 0.846 & 1.000 & $89.39 \%$ & 0.842 & 0.964 & $83.33 \%$ & 0.750 & 1.000 \\
\hline 23 & $63.64 \%$ & 0.712 & 0.636 & $43.94 \%$ & 0.500 & 0.468 & $48.48 \%$ & 0.400 & 0.492 \\
\hline 24 & $62.12 \%$ & 0.700 & 0.841 & $63.64 \%$ & 0.580 & 0.840 & $50.00 \%$ & 0.500 & 0.500 \\
\hline 25 & $50.00 \%$ & 0.500 & 0.500 & $57.58 \%$ & 0.667 & 0.549 & $83.33 \%$ & 1.000 & 0.502 \\
\hline 26 & $89.39 \%$ & 0.842 & 0.964 & $57.58 \%$ & 0.600 & 0.610 & $66.67 \%$ & 0.600 & 0.814 \\
\hline & $\mathbf{6 0 . 0 7}$ & $\mathbf{0 . 6 2 4}$ & $\mathbf{0 . 6 0 5}$ & $\mathbf{5 6 . 9 5}$ & $\mathbf{0 . 6 1 0}$ & $\mathbf{0 . 5 6 6}$ & $\mathbf{5 7 . 5 8}$ & $\mathbf{0 . 6 4 0}$ & $\mathbf{0 . 5 9 9}$ \\
\hline
\end{tabular}

En cuestión de precisiones, las mejores se presentan en la clase de arriba porque en cada una de las combinaciones donde está presente, su precisión está por encima de 0.60. La transferencia de aprendizaje con mayor deficiencia se observa en el sujeto 2 con algunas precisiones iguales a cero. En lo que concierne a la combinación de las clases, las de mayor precisión son arriba-abajo y arriba-izquierda. Por otro lado, se tiene en caso de las clases arriba-izquierda en donde se encuentra la precisión promedio más baja (izquierda $=0.492$ ). 
Tabla 2. Resultado de 3 combinaciones entre clases.

\begin{tabular}{|c|c|c|c|c|c|c|c|c|c|}
\cline { 2 - 10 } \multicolumn{1}{c|}{} & \multicolumn{2}{|c|}{ DERECHA-IZQUIERDA } & \multicolumn{2}{c|}{ ABAJO-DERECHA } & \multicolumn{2}{c|}{ ABAJO-IZQUIERDA } \\
\hline $\begin{array}{c}\text { Sujeto } \\
\text { s }\end{array}$ & EXAC & $\begin{array}{c}\text { Precisió } \\
\mathrm{n} \\
\text { derecha }\end{array}$ & $\begin{array}{c}\text { Precisió } \\
\mathrm{n} \\
\text { izquierd } \\
\mathrm{a}\end{array}$ & EXAC. & $\begin{array}{c}\text { Precisió } \\
\mathrm{n} \\
\text { abajo }\end{array}$ & $\begin{array}{c}\text { Precisió } \\
\mathrm{n} \\
\text { derecha }\end{array}$ & EXAC. & $\begin{array}{c}\text { Precisió } \\
\mathrm{n} \\
\text { abajo }\end{array}$ & $\begin{array}{c}\text { Precisió } \\
\mathrm{n} \\
\text { izquierd } \\
\mathrm{a}\end{array}$ \\
\hline 2 & $50.00 \%$ & 0.500 & 0.000 & $50.00 \%$ & 0.500 & 0.000 & $50.00 \%$ & 0.500 & 0.500 \\
\hline 3 & $59.09 \%$ & 0.684 & 0.645 & $63.64 \%$ & 0.615 & 0.575 & $50.00 \%$ & 0.500 & 0.500 \\
\hline 6 & $59.09 \%$ & 0.750 & 0.556 & $46.97 \%$ & 0.333 & 0.483 & $43.94 \%$ & 0.500 & 0.250 \\
\hline 7 & $54.55 \%$ & 0.552 & 0.541 & $48.48 \%$ & 0.490 & 0.471 & $37.88 \%$ & 0.357 & 0.578 \\
\hline 10 & $42.42 \%$ & 0.381 & 0.444 & $50.00 \%$ & 0.500 & 0.500 & $60.61 \%$ & 0.585 & 0.640 \\
\hline 12 & $89.39 \%$ & 0.574 & 0.812 & $59.09 \%$ & 0.526 & 0.667 & $60.62 \%$ & 0.585 & 0.756 \\
\hline 13 & $63.64 \%$ & 0.570 & 0.700 & $50.00 \%$ & 0.500 & 0.500 & 100.00 & 1.000 & 0.000 \\
\hline 14 & $63.64 \%$ & 0.800 & 0.588 & $42.42 \%$ & 0.381 & 0.444 & $46.97 \%$ & 0.480 & 0.438 \\
\hline 16 & $59.09 \%$ & 0.571 & 0.751 & $59.09 \%$ & 0.845 & 0.610 & $60.62 \%$ & 0.612 & 0.712 \\
\hline 17 & $59.09 \%$ & 0.570 & 0.611 & $59.09 \%$ & 0.575 & 0.615 & $46.97 \%$ & 0.462 & 0.475 \\
\hline 19 & $45.45 \%$ & 0.472 & 0.385 & $54.55 \%$ & 0.526 & 0.667 & $48.48 \%$ & 0.444 & 0.491 \\
\hline 21 & $63.64 \%$ & 0.528 & 0.615 & $62.62 \%$ & 0.754 & 0.700 & $48.48 \%$ & 0.474 & 0.489 \\
\hline 22 & $59.09 \%$ & 0.588 & 0.594 & $40.91 \%$ & 0.842 & 0.446 & $57.58 \%$ & 0.551 & 0.647 \\
\hline 23 & $51.52 \%$ & 0.571 & 0.508 & $50.00 \%$ & 0.500 & 0.500 & $39.39 \%$ & 0.431 & 0.267 \\
\hline 24 & $63.63 \%$ & 0.800 & 0.712 & $63.64 \%$ & 0.512 & 0.600 & $57.58 \%$ & 0.550 & 0.641 \\
\hline 25 & $59.09 \%$ & 0.512 & 0.754 & $42.42 \%$ & 0.459 & 0.450 & $43.94 \%$ & 0.463 & 0.333 \\
\hline 26 & $63.64 \%$ & 0.514 & 0.700 & $59.09 \%$ & 0.526 & 0.662 & $60.62 \%$ & 0.585 & 0.640 \\
\hline & $\mathbf{5 9 . 1 8}$ & $\mathbf{0 . 5 8 5}$ & $\mathbf{0 . 5 8 3}$ & $\mathbf{5 3 . 0 6}$ & $\mathbf{0 . 5 5 2}$ & $\mathbf{0 . 5 2 3}$ & $\mathbf{5 3 . 7 5 \%}$ & $\mathbf{0 . 5 3 4}$ & $\mathbf{0 . 4 9 2}$ \\
\hline
\end{tabular}

\section{Conclusiones y trabajo futuro}

El presente trabajo describe un método que abarcaría los primeros pasos hacia un método transferencia de aprendizaje sujeto-a-sujeto en señales de EEG en habla imaginada. Los experimentos realizados se orientaron a observar si los datos de entrenamiento adquiridos para otros sujetos pueden ser utilizados como datos de entrenamiento para un nuevo sujeto, sin incluir ningún tipo de información o calibración.

De los resultados obtenidos y mostrados en la sección anterior, se observa que el porcentaje de exactitud entre dos clases es diferente en cada combinación, y eso nos indica que hay clases que son más diferenciables de otras, por ejemplo, la clase con mayor exactitud promedio es arriba-abajo con el $60.07 \%$, seguida de las clases derecha- 
izquierda con $59.18 \%$. Por otro lado, tenemos que las clases menos diferenciables y en la que las instancias se confunden más son las clases abajo-izquierda y abajo-derecha.

No obstante, las tasas de exactitud promedio están entre $53 \%$ y $60 \%$, que aunque lejos de resolver el problema, son resultados muy alentadores. Sobre todo porque en estos primeros experimentos no se recurrió a ningún proceso de adaptación hacia los nuevos sujetos. De hecho, se llegó a tener casos con exactitudes promedio similares a los reportados en el estado del arte [5].

Por otro lado, existe una fuerte variación de los clasificadores entre los sujetos. Se tiene el caso de los sujetos 22 y 26 que presentan altas exactitudes en todos los clasificadores; pero también se presenta el caso del sujeto 2 con muy bajos resultados.

Como trabajo futuro se desean explorar varias ideas. Por un lado, utilizar otro método para seleccionar las instancias de entrenamiento, al menos para aquellas clases en que no se tienen información para discriminar correctamente entre ellas. Por otro lado, incluir un módulo de adaptación o calibración que permita incluir información del nuevo sujeto. Para ello se pretende seguir lo propuesto en [6]. Donde como primer paso para adaptar los datos de cada EEG de un sujeto a una base de datos base de EEG, se minimiza la distribución entre los dos conjuntos de EEG.

Agradecimientos. Los autores agradecen al Consejo Nacional de Ciencia y Tecnología (CONACyT) por el apoyo a esta investigación, el cual se realizó a través de la beca 701206 y del proyecto No. Ref. 2016-01-2228. Los autores también desean agradecer el apoyo de la Red Temática en Tecnologías del Lenguaje/CONACYT, No. Ref. 260178, 271622, por los mecanismos de colaboración brindados para realizar este trabajo. De igual manera, agradecemos a la Vicerrectoría de Investigación y Estudios de Posgrado (VIEP) por el apoyo brindado para la publicación del presente trabajo.

\section{Bibliografía}

1. Morales-Flores, E., Ramírez-Cortés, J. M., Gómez-Gil, P., Alarcón-Aquino, V.: Brain Computer Interface Development Based on Recurrent Neural Networks and ANFIS Systems. Springer, pp. 215-236 (2013)

2. Torres-García, A., Reyes-García, C. A., Villaseñor-Pineda, L., Ramírez-Cortés, J. M.: Análisis de Señales Electroencefalográficas para la Clasificación de Habla Imaginada. Revista Mexicana Ingenería Biomédica, pp. 23-29 (2013)

3. Jayaram, V., Alamgir, M., Altun, Y., Schökopf, B.: Wentrup G.: Transfer Leaning in BrainComputer Interfaces. IEEE, pp. 20-31 (2016)

4. Kang, H., Nam, Y., Choi, S.: Composite Common Spatial Pattern for Subject-to-Subject Transfer. IEEE Signal Processing Letters, pp. 683-686 ( 2009)

5. Hyun-Lee, S., Abibullaev, B., Kang, W. S., Shin, Y., An, J.: Analysis of Attention Deficit Hyperactivity Disorder in EEG Using Wavelet Transform and Self Organizing Maps. In: International Conference on Control, Automation and Systems, pp. 2439-2442 ( 2010)

6. Arvaneh, M., Robertson, I., Ward, T. E.: Subject-to-Subject Adaptation to Reduce Calibration Time in Motor Imagery-based Brain-Computer Interface. IEEE, pp. 6501-6504 (2014)

7. Marioka, H., Kanemura, A., Hiramaya, J. I., Shikauchi, M., Ogawa, T., Ikeda, S., Kawanabe, M., Ishii, S.: Learning a common dictionary for subject-transfer decoding with resting calibration. NeuroImage, pp. 167-178 (2015) 